\title{
GMR
}

\section{GRK2 desensitizes flow-induced responses in osteoblasts}

\author{
Y. Xing ${ }^{1}$, Y. Gu ${ }^{2}$, X. Shan ${ }^{1}$, L. Wang ${ }^{1}$ and J. You ${ }^{2}$ \\ ${ }^{1}$ Beijing Key Laboratory of Rehabilitation Technical Aids for Old-Age Disability, \\ National Research Center for Rehabilitation Technical Aids, Beijing, China \\ ${ }^{2}$ Department of Orthopaedics and Rehabilitation, \\ The Pennsylvania State University College of Medicine, Hershey, PA, USA \\ Corresponding author: Y. Xing \\ E-mail: xingyanghui@hotmail.com \\ Genet. Mol. Res. 16 (1): gmr16019363 \\ Received September 21, 2016 \\ Accepted September 21, 2016 \\ Published January 23, 2017 \\ DOI http://dx.doi.org/10.4238/gmr16019363
}

Copyright $(2017$ The Authors. This is an open-access article distributed under the terms of the Creative Commons Attribution ShareAlike (CC BY-SA) 4.0 License.

\begin{abstract}
Bone desensitization after mechanical loading is essential for bone to adapt to its mechanical environment. However, the desensitization mechanism is unknown. Previous studies suggest that G protein-coupled receptors (GPCRs), including P2Y and parathyroid hormone receptors, play important roles in osteoblast mechanobiology. Thus, for the present research, we examined the role of G protein-coupled receptor kinase 2 (GRK2) in osteoblast desensitization after exposure to mechanical stimulation. We first showed the existence of osteoblast desensitization after mechanical stimulation based on cytosol $\mathrm{Ca}^{2+}$ and phosphorylated ERK1/2 activities, detected using a fluorescent $\mathrm{Ca}^{2+}$ sensitive dye and western blotting, respectively. We then demonstrated that GRK2 overexpression in MC3T3-E1 cells inhibits flow-induced ERK1/2 phosphorylation, while siRNA knockdown of GRK2 enhances ERK1/2 phosphorylation. Additionally, we found that GRK2 overexpression in MC3T3-E1 cells inhibits cyclooxygenase-2 mRNA
\end{abstract}


expression in the short term and alkaline phosphatase activity in the long term. More importantly, we discovered that GRK2 translocated to the cell membrane shortly after flow stimulation - a step necessary for GPCR desensitization. Previously, we have demonstrated that P2Y2 purinergic receptors, one type of GPCRs, are involved in various flowinduced osteoblastic responses. In this research, we also showed that GRK2 overexpression does not affect ATP release. Accordingly, GRK2 is able to inhibit flow-induced osteoblast responses possibly through desensitizing P2Y2 receptors.

Key words: Osteoblast; GRK2; Bone desensitization; Oscillatory flow; Mechanotransduction

\section{INTRODUCTION}

External loadings that induce biophysical signals have been demonstrated to regulate bone cell activities, making them respond with the release of signaling molecules and alterations in gene expression (Klein-Nulend et al., 2005; Robling et al., 2006; Papachroni et al., 2009). However, the mechanisms underlying the initiation and execution of these cellular responses have not been elucidated. Accumulating evidence suggests that oscillatory fluid flow is a very strong initiator for bone cell metabolic activities, including cell growth and differentiation, gene expression, and protein production (You et al., 2001, 2002; Ponik et al., 2007; Xing et al., 2014). In an earlier study, we demonstrated the role of oscillatory flow in the induction of cytosol $\mathrm{Ca}^{2+}$ release from the ER, and enhancement of mRNA expression of osteopontin (You et al., 2001). Other researchers also showed that flow stimulation on bone cells enhances cyclooxygenase-2 (COX-2) expression, causes activation of the ERK pathway, and leads to ATP release (Wadhwa et al., 2002; Genetos et al., 2007; Okumura et al., 2008). However, long-term constant mechanical loading does not increase the response of bone cells continuously. Based on earlier reports, there is a strong indication that the bone cells become unresponsive rapidly by a negative feedback mechanism such as receptor desensitization or increasing the threshold for a second stimulation (Robling et al., 2002; LaMothe and Zernicke, 2004). Such desensitization of bone after mechanical loading is essential for bone to adapt to its mechanical environment, but it still remains unclear how the bone become desensitized.

Various G protein-coupled receptors (GPCRs) are involved in bone mechanobiology, such as P2Y purinergic receptors and parathyroid hormone (PTH) receptors (Xing et al., 2014; Gardinier et al., 2014). Previously, we found that P2Y2 receptors play an important role in ERK phosphorylation and $\mathrm{Ca}^{2+}$ mobilization in osteoblast response to oscillatory fluid flow (You et al., 2002). Other studies have shown P2Y1, P2Y6, and P2Y13 are also related to bone phenotype changes (Orriss et al., 2011). Additionally, the PTH receptor, another GPCR, is able to affect bone metabolism synergistically with mechanical stimulation (Maycas et al., 2015; Falk et al., 2016). GPCRs are not always active, and they may be desensitized by a group of GPCR kinases (GRKs) with the assistance of arrestins (Pitcher et al., 1998; Kohout and Lefkowitz, 2003). Desensitizing GPCRs is a crucial action performed by bone cells in order to maintain their normal functions (Turner, 1998; Gross et al., 2004). A significant amount of GRK2, a subgroup of GRKs, exists in skeletal systems; and a previous study demonstrated that inhibiting GRK phosphorylation enhanced PTH receptor-stimulated cAMP generation

Genetics and Molecular Research 16 (1): gmr16019363 
in osteoblastic cells, suggesting that GRKs may be involved in desensitization of the PTH receptor (Wang et al., 2004). Nevertheless, the mechanism of GRK2 desensitization of osteoblasts due to mechanical stimulation remains elusive.

To study the mechanism, MC3T3-E1 osteoblasts and oscillatory fluid flow were used to examine whether GRK2 is involved in bone cell desensitization after mechanical stimulation. We used ERK1/2 phosphorylation as an indicator to test osteoblast bioactivities after exposure to flow stimulation. Because the active phosphorylated ERK (P-ERK) influences many important osteoblast bioactivities, such as proliferation and differentiation, it was used as one of the key indicators to test the effects of mechanical stimulation. We will also examine COX-2 expression and alkaline phosphatase (AP) activities, which are mid-term and long-term indicators of osteoblast metabolism, respectively. In this study, we first examined the existence of osteoblast desensitization after mechanical stimulation. We then studied the role of GRK2 in osteoblast responses to elucidate the mechanism of osteoblast desensitization.

\section{MATERIAL AND METHODS}

\section{Oscillatory fluid flow and cell culture}

Murine osteoblasts (MC3T3-E1 subclone 14) were cultured in minimal essential medium $\alpha$ supplemented with $10 \%$ FBS and 1\% penicillin-streptomycin under standard cell culture conditions. For subsequent experiments, osteoblasts were split on glass slides for two days before experiments. Quartz slides with good UV transparency were used for $\mathrm{Ca}^{2+}$ imaging based on our existing protocols. Cells were exposed to $10 \mathrm{dyne} / \mathrm{cm}^{2}, 1 \mathrm{~Hz}$, sinusoidal pattern, oscillatory fluid flow with minimal essential medium $\alpha$ and $2 \% \mathrm{FBS}$ for $\mathrm{Ca}^{2+}$ imaging experiments. Different concentrations of FBS were selected to optimize fluid flow responses to $\mathrm{Ca}^{2+}$ mobilization and ERK1/2 activation (You et al., 2001; Xing et al. 2014).

\section{Intracellular $\mathrm{Ca}^{2+}$ release quantification}

We used a well-established method to quantify $\mathrm{Ca}^{2+}$ release in osteoblasts when experiencing mechanical stimulation, as reported in our early research (You et al., 2001; Xing et al. 2014). Briefly, the intracellular $\mathrm{Ca}^{2+}$ concentration was measured with the use of the fluorescent dye fura-2, which binds $\mathrm{Ca}^{2+}$. When osteoblasts were exposed to UV under this condition, there is a change in emission intensity at different wavelengths - when there is more $\mathrm{Ca}^{2+}$, we see an increase at $340 \mathrm{~nm}$, but a decrease at $380 \mathrm{~nm}$. The ratio of light intensity between the two wavelengths represents the intracellular $\mathrm{Ca}^{2+}$ release.

\section{Overexpression and siRNA knockdown of GRK2}

To overexpress GRK2 in cells, full length GRK2 cDNA plasmid (donated by Professor RT Premont; Liu et al., 2005) was transfected into MC3T3-E1 osteoblasts using the FuGENE 6 transfection reagent kit from Roche (Basel, Switzerland) based on their official protocols. For siRNAs directly against GRK2, si-GRK2 sense/antisense was designed and manufactured by Qiagen, Inc. (Hilden, Germany). The efficiency of knocking down was confirmed by mRNA and protein expression using RT-PCR and western blotting respectively.

Genetics and Molecular Research 16 (1): gmr16019363 


\section{Membrane protein extraction and western blot}

Cell membrane proteins were extracted using a commercially available Mem-PER Plus Membrane Protein Extraction kit from Pierce Biotechnology, Inc. (Thermo-Fisher Scientific, Waltham, MA, USA) based on protocols provided by the manufacturer. Western blot experiments were carried out using our existing protocols (Xing et al., 2011a; 2014). Briefly, cytosol proteins were extracted with cell lysis buffer, then protein concentrations were measured by using a protein assay kit from Pierce Biotechnology, Inc. Proteins were electrophoresed in SDS-PAGE gel, then a PVDF membrane was used for protein transfer. For phosphorylated ERK1/2 expression, total ERK1/2 and GAPDH were used as control proteins. For membrane GRK2 expression the total protein concentration served as a control, verified by Coomassie blue staining and GAPDH expression. Quantification of western blot analysis was carried out with Bio-Rad GS-800 densitometer (Bio-Rad Laboratories, Inc., Hercules, CA, USA) and the Quality One image analysis software (Bio-Rad Laboratories).

\section{AP and ATP evaluation}

To examine AP activity, cells were rinsed two times with phosphate-buffered saline, then freeze-thawed three times at $-80^{\circ} \mathrm{C}$ and room temperature, respectively, to break the cell membrane. Subsequently, the measurement of AP activities was performed according to our previous protocols (Xing et al., 2014). To examine ATP concentration, we first collected conditioned media during oscillatory flow stimulation, and then stored it at $-80^{\circ} \mathrm{C}$ until the following experiment: ATP concentration was measured with the Roche ATP bioluminescence kit. For the details of ATP measurement, please refer our early studies (Xing et al., 2011b).

\section{Statistics}

Experimental results were tested with the statistical software MINITAB (Minitab, Inc., State College, PA, USA). Data are reported as means \pm SE. The two-sample Students $t$-test was used to examine the difference between groups. For our purpose, we only compared different groups exposed to mechanical stimulation. An asterisk on the bar graph denotes a $\mathrm{P}$ value smaller than 0.05 , which implies a significant difference in statistics.

\section{RESULTS}

\section{$\mathrm{Ca}^{2+}$ and P-ERK1/2 desensitization after mechanical stimulation}

We first examined intracellular $\mathrm{Ca}^{2+}$ mobilization in response to two 3-min bouts of oscillatory fluid flow separated by a 5-min rest period (Figure 1A). The peak shear stress of oscillatory fluid flow was 10 dynes $/ \mathrm{cm}^{2}$ at $1 \mathrm{~Hz}$ frequency. MC3T3-E1 cells exhibited desensitization in terms of the $\mathrm{Ca}^{2+}$ response during the second oscillatory fluid flow period. Both cells responding to fluid flow and the mean $\mathrm{Ca}^{2+}$ response amplitude during the second flow period were decreased significantly, by about $50 \%$, compared with those in the first flow period (Figure 1B).

We also examined the influence of intermittent fluid flow $\left(10\right.$ dynes $\left./ \mathrm{cm}^{2}, 1 \mathrm{~Hz}\right)$ on ERK1/2 activation. Based on previous ERK1/2 experiments, we used a protocol that includes two 5-min bouts of oscillatory fluid flow separated by a 20 -min rest period. Our results 
demonstrated that the ERK1/2 response during the second flow period was significantly decreased compared with that during the first flow period (Figure 1C and D). Thus, our results clearly demonstrated the desensitization of osteoblasts after mechanical stimulation in vitro.

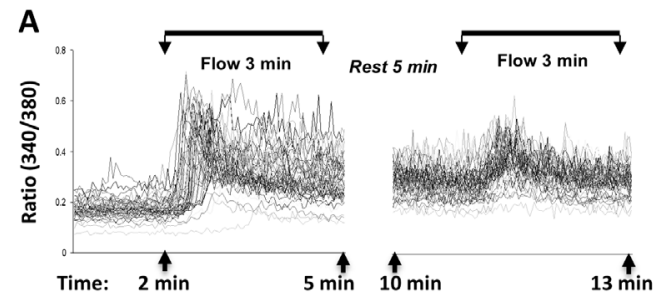

B
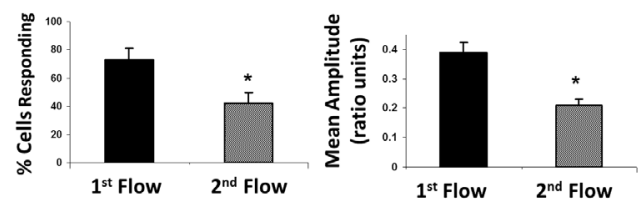

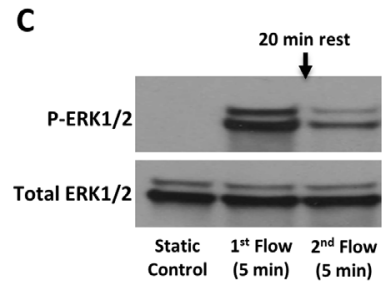

D

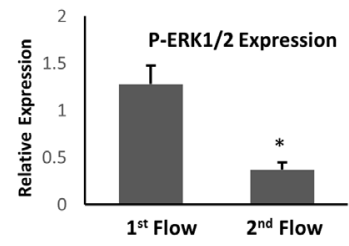

Figure 1. A. $\mathrm{Ca}^{2+}$ imaging of osteoblasts in response to intermittent flow stimuli. B. Statistical analysis of $\mathrm{Ca}^{2+}$ imaging results. C. Phosphorylated ERK protein expression after fluid flow. D. Statistical analysis of relative phosphorylated ERK1/2 expression normalized to total ERK1/2 expression $(\mathrm{N}=3)$.

\section{GRK2 in osteoblast P-ERK desensitization}

GPRCs including P2Y and PTH receptors are involved in bone mechanotransduction. All GPCRs undergo desensitization by GRKs, and GRK2 is a major GRK expressed in osteoblasts. To elucidate the role of GRK2 in osteoblast mechanobiology, we further examined ERK activation in response to flow stimulation in MC3T3-E1 cells. The experimental results showed that the ERK phosphorylation level is significantly decreased after GRK2 overexpression (Figure 2A). GRK2-K220R is a dominant negative mutant of GRK2. When we overexpressed this specific mutant in MC3T3-E1 cells, the phosphorylation level of ERK1/2 did not have a significant change under fluid flow stimulation compared with vector control samples (Figure 2A). Additionally, after siRNA knockdown of the GRK2 level, we observed a significant boost in phosphorylated ERK1/2 expression after mechanical stimulation (Figure 2B). The results suggest that GRK2 inhibits flow-induced ERK phosphorylation in osteoblasts.

\section{GRK2 in COX-2 and AP activity desensitization}

Prostaglandins are important mediators of bone repair and COX-2 is required for prostaglandin production. To further investigate the mechanism of how GRK2 desensitizes osteoblasts, we also checked the cell COX-2 mRNA level before and after fluid shear stimulation using vector and GRK2 plasmid transfection. We found that GRK2 inhibits COX2 mRNA expression significantly for 1, 2, and $4 \mathrm{~h}$ of flow stimulation (Figure 3A). Next, we examined the effect of GRK2 on regulating AP activities in MC3T3-E1 osteoblastic cells. AP is an enzyme that is important for new bone formation. We found AP activities of MC3T3-E1 cells increased slowly in the first two weeks; there is then an 11-fold increase at day 25. 
MC3T3-E1 cells transfected with GRK2 have significantly decreased AP activities compare to cells transfected with the vector only (Figure 3B). These results further demonstrate that GRK2 is involved in osteoblast desensitization after mechanical stimulation.

A

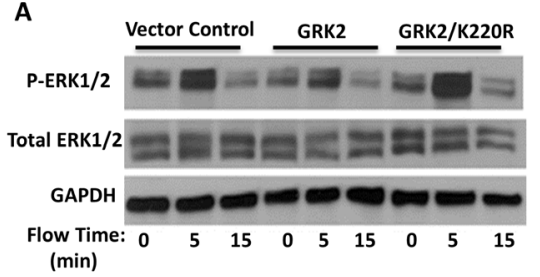

B

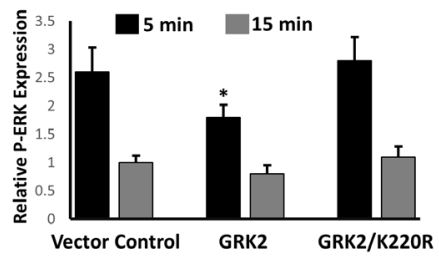

C

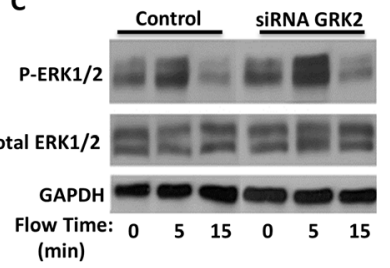

D

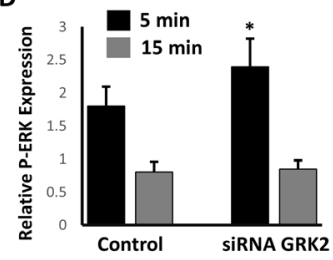

Figure 2. A. C. Western blot images showing the ERK1/2 phosphorylation level after oscillatory fluid flow stimulation with GRK2, GRK2-K220R overexpression, and GRK2 siRNA knockout. B. D. Statistical analyses of P-ERK1/2 expressions normalized to total ERK1/2 expressions $(\mathrm{N}=4)$.

A

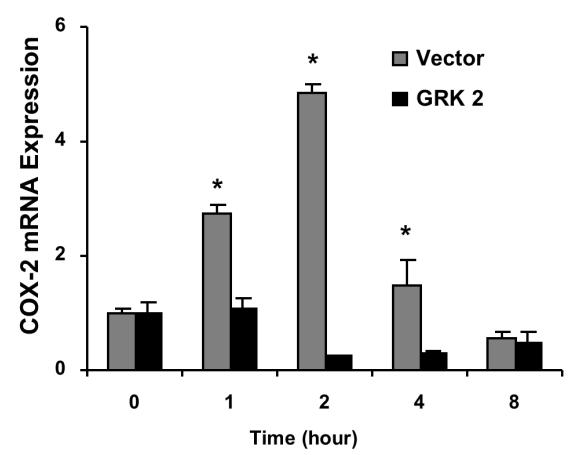

B

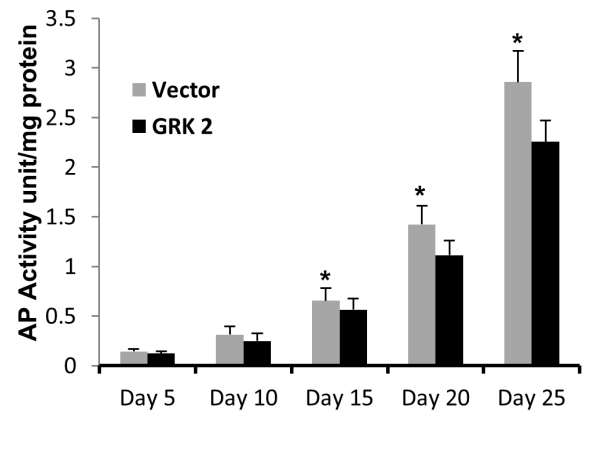

Figure 3. A. Bar graph representation of the mRNA level of COX-2 normalized to GAPDH expression. B. Bar graph of quantified AP activity normalized to total protein concentration $(\mathrm{N}=3)$.

\section{GRK2 translocation after mechanical stimulation}

GRK2 is mainly expressed in the intracellular space, while GPCRs are membrane proteins. In order to desensitize GPCRs, GRK2 has to move to the cell membrane. Therefore, we further investigated GRK2 movement in response to flow stimulation. We found that after mechanical stimulation, GRK2 translocated to the cell membrane rapidly. The result suggests mechanical stimulation is able to activate GRK2 to phosphorylate membrane GPCRs (Figure 
4A). We also found that GRK2 overexpression does not alter ATP release after mechanical stimulation (Figure 4B), suggesting that GRK2 may inhibit P2Y receptors directly instead of by regulating ATP release.

A

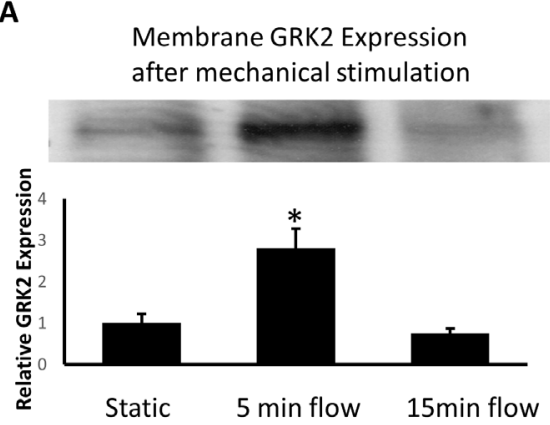

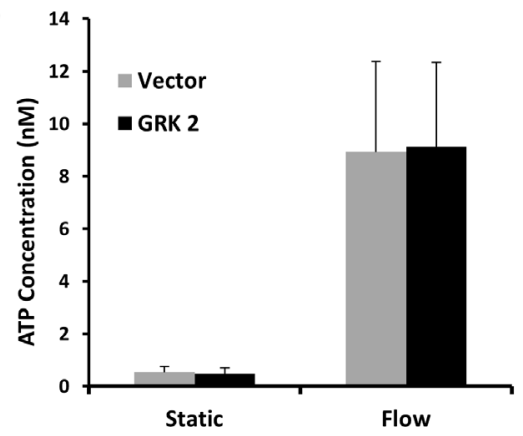

Figure 4. A. Membrane GRK2 protein expression after oscillatory fluid flow stimulation. The statistical analysis GRK2 expression was normalized to total protein concentration. B. Bar graph representation of ATP concentrations in conditioned media after 1 minute of flow $(\mathrm{N}=3)$.

\section{DISCUSSION}

Mechanical loadings regulate bone growth and remodeling, but continuous mechanical stimulation will not lead to continuous cell responses such as ATP release, phosphorylation of ERK1/2, and cytosol $\mathrm{Ca}^{2+}$ activity. Usually, cell response is the strongest in the first several minutes, and extra mechanical stimulation causes a much weaker response than initially because of bone desensitization (Robling et al., 2002; LaMothe and Zernicke, 2004). At the cellular level, mechanical stimulation is able to induce various responses such as ATP and $\mathrm{Ca}^{2+}$ release and ERK phosphorylation in osteoblasts in a few minutes. Many of these responses decrease quickly and significantly from their maximum points to a relatively low level. In this study, we corroborated that a second mechanical stimulation, following a brief rest after the first stimulation, causes a much weaker cell response compared to the first one. Our results also suggest that osteoblasts desensitize after the initial mechanical stimulation.

Mechanical loading induces ATP release in osteoblastic cells. In turn, ATP activates P2Y purinergic signaling pathways (Ayala-Peña et al., 2013; Rodrigues-Ribeiro et al., 2015). We also used external ATP stimulation to replace oscillatory fluid flow, and we obtained similar results for all our experiments; the results of ERK activation by fluid flow or ATP confirm the important role of ATP release in bone cell mechanotransduction. Overexpression of P2Y2 enhances the phosphorylation of ERK in response to both fluid flow and ATP stimulation, while the siRNA knockdown of P2Y2 inhibits ERK phosphorylation. In addition, P2Y2 is also responsible for mechanical loading-induced $\mathrm{Ca}^{2+}$ release in osteoblasts (Xing et al., 2014; Gardinier et al., 2014). These results demonstrate that P2Y2 mechanotransduction pathway is of great importance in bone cell mechanobiology. Thus, P2Y2 may also experience desensitization after mechanical stimulation.

P2Y purinergic receptors are GPCRs. GPCRs are desensitized and regulated by GRKs, and GRK2 is the major GRK expressed in bone (Bliziotes et al., 2000). Our results of overexpressing GRK2 demonstrated that GRK2 is involved in the desensitization of ERK 
activation stimulated by both fluid flow and external ATP. The result suggests that GRK2 may inhibit the ERK activation by desensitizing P2Y receptors. GRK2-K220R is the kinase deficient mutant of GRK2. When we overexpressed this specific mutant, the phosphorylation of ERK does not change. This suggests that the kinase activity of GRK2 is necessary for P2Y2 desensitization and K220 is the critical site for GRK2 functionality to desensitize P2Y receptors.

GPCR desensitization needs GRK translocation to the plasma membrane and the formation of protein complexes on the membrane. Our translocation results showed that upon ATP stimulation, the amount of GRK2 on the cell membrane was increased significantly suggesting the possible formation of $\mathrm{P} 2 \mathrm{Y} / \mathrm{GRK} 2$ protein complexes on the membrane. This strengthens the functional role of GRK2 in desensitizing P2Y receptors.

Taken together, our data demonstrate that GRK2 suppresses ERK1/2 activities caused by mechanical stimuli, in MC3T3-E1 cells, possibly through P2Y2 receptors, and suggest that GRK2 may have important functions in modulating mechano-desensitization in osteoblastic cells.

\section{Conflicts of interest}

The authors declare no conflict of interest.

\section{ACKNOWLEDGMENTS}

Research supported by the National Institutes of Health Grants \#AR054851 (awarded to J. You) and the National Natural Science Foundation of China (\#11672078, awarded to Y. Xing). We greatly appreciate the gift of mouse GRK2 in pcDNA3 plasmid from Dr. Richard T. Premont (Duke University, Durham, NC, USA).

\section{REFERENCES}

Ayala-Peña VB, Scolaro LA and Santillán GE (2013). ATP and UTP stimulate bone morphogenetic protein-2,-4 and -5 gene expression and mineralization by rat primary osteoblasts involving PI3K/AKT pathway. Exp. Cell Res. 319: 2028-2036. http://dx.doi.org/10.1016/j.yexcr.2013.05.006

Bliziotes M, Gunness M, Zhang X, Nissenson R, et al. (2000). Reduced G-protein-coupled-receptor kinase 2 activity results in impairment of osteoblast function. Bone 27: 367-373. http://dx.doi.org/10.1016/S8756-3282(00)00338-0

Falk B, Haddad F, Klentrou P, Ward W, et al. (2016). Differential sclerostin and parathyroid hormone response to exercise in boys and men. Osteoporos. Int. 27: 1245-1249. http://dx.doi.org/10.1007/s00198-015-3310-z

Gardinier J, Yang W, Madden GR, Kronbergs A, et al. (2014). P2Y2 receptors regulate osteoblast mechanosensitivity during fluid flow. Am. J. Physiol. Cell Physiol. 306: C1058-C1067.http://dx.doi.org/10.1152/ajpcell.00254.2013

Genetos DC, Kephart CJ, Zhang Y, Yellowley CE, et al. (2007). Oscillating fluid flow activation of gap junction hemichannels induces ATP release from MLO-Y4 osteocytes. J. Cell. Physiol. 212: 207-214. http://dx.doi.org/10.1002/jcp.21021

Gross TS, Poliachik SL, Ausk BJ, Sanford DA, et al. (2004). Why rest stimulates bone formation: a hypothesis based on complex adaptive phenomenon. Exerc. Sport Sci. Rev. 32: 9-13.http://dx.doi.org/10.1097/00003677-200401000-00003

Klein-Nulend J, Bacabac RG and Mullender MG (2005). Mechanobiology of bone tissue. Pathol. Biol. (Paris) 53: 576580.http://dx.doi.org/10.1016/j.patbio.2004.12.005

Kohout TA and Lefkowitz RJ (2003). Regulation of G protein-coupled receptor kinases and arrestins during receptor desensitization. Mol. Pharmacol. 63: 9-18.http://dx.doi.org/10.1124/mol.63.1.9

LaMothe JM and Zernicke RF (2004). Rest insertion combined with high-frequency loading enhances osteogenesis. $J$. Appl. Physiol. (1985). 96: 1788-93.

Liu S, Premont RT, Kontos CD, Zhu S, et al. (2005). A crucial role for GRK2 in regulation of endothelial cell nitric oxide synthase function in portal hypertension. Nat. Med. 11: 952-958. http://dx.doi.org/10.1038/nm1289

Maycas M, Ardura JA, de Castro LF, Bravo B, et al. (2015). Role of the Parathyroid Hormone Type 1 Receptor (PTH1R) as a Mechanosensor in Osteocyte Survival. J. Bone Miner. Res. 30: 1231-1244.http://dx.doi.org/10.1002/jbmr.2439

Okumura H, Shiba D, Kubo T and Yokoyama T (2008). P2X7 receptor as sensitive flow sensor for ERK activation in

Genetics and Molecular Research 16 (1): gmr16019363 
GRK2 desensitizes flow-induced responses in osteoblasts

osteoblasts. Biochem. Biophys. Res. Commun. 372: 486-490. http://dx.doi.org/10.1016/j.bbrc.2008.05.066

Orriss I, Syberg S, Wang N, Robaye B, et al. (2011). Bone phenotypes of P2 receptor knockout mice. Front. Biosci. (Schol. Ed.) 3: 1038-1046. http://dx.doi.org/10.2741/208

Papachroni KK, Karatzas DN, Papavassiliou KA, Basdra EK, et al. (2009). Mechanotransduction in osteoblast regulation and bone disease. Trends Mol. Med. 15: 208-216. http://dx.doi.org/10.1016/j.molmed.2009.03.001

Pitcher JA, Freedman NJ and Lefkowitz RJ (1998). G protein-coupled receptor kinases. Anпи. Rev. Biochem. 67: 653-692. http://dx.doi.org/10.1146/annurev.biochem.67.1.653

Ponik SM, Triplett JW and Pavalko FM (2007). Osteoblasts and osteocytes respond differently to oscillatory and unidirectional fluid flow profiles. J. Cell. Biochem. 100: 794-807. http://dx.doi.org/10.1002/jcb.21089

Robling AG, Hinant FM, Burr DB and Turner CH (2002). Improved bone structure and strength after long-term mechanical loading is greatest if loading is separated into short bouts. J. Bone Miner. Res. 17: 1545-1554. http:// dx.doi.org/10.1359/jbmr.2002.17.8.1545

Robling AG, Castillo AB and Turner CH (2006). Biomechanical and molecular regulation of bone remodeling. Annu. Rev. Biomed. Eng. 8: 455-498. http://dx.doi.org/10.1146/annurev.bioeng.8.061505.095721

Rodrigues-Ribeiro R, Alvarenga ÉC, Calio ML, Paredes-Gamero EJ, et al. (2015). Dual role of P2 receptors during osteoblast differentiation. Cell Biochem. Biophys. 71: 1225-1233. http://dx.doi.org/10.1007/s12013-014-0332-7

Turner CH (1998). Three rules for bone adaptation to mechanical stimuli. Bone 23: 399-407. http://dx.doi.org/10.1016/ $\underline{\mathrm{S} 8756-3282(98) 00118-5}$

Wadhwa S, Godwin SL, Peterson DR, Epstein MA, et al. (2002). Fluid flow induction of cyclo-oxygenase 2 gene expression in osteoblasts is dependent on an extracellular signal-regulated kinase signaling pathway. J. Bone Miner. Res. 17: 266-274. http://dx.doi.org/10.1359/jbmr.2002.17.2.266

Wang L, Quarles LD and Spurney RF (2004). Unmasking the osteoinductive effects of a G-protein-coupled receptor (GPCR) kinase (GRK) inhibitor by treatment with PTH(1-34). J. Bone Miner. Res. 19: 1661-1670. http://dx.doi. org/10.1359/JBMR.040708

Xing Y, Gu Y, Gomes RR, Jr. and You J (2011). P2Y(2) receptors and GRK2 are involved in oscillatory fluid flow induced ERK1/2 responses in chondrocytes. J. Orthop. Res. 29: 828-833. http://dx.doi.org/10.1002/jor.21319

Xing Y, Gu Y, Xu LC, Siedlecki CA, et al. (2011). Effects of membrane cholesterol depletion and GPI-anchored protein reduction on osteoblastic mechanotransduction. J. Cell. Physiol. 226: 2350-2359. http://dx.doi.org/10.1002/jcp.22579

Xing Y, Gu Y, Bresnahan JJ, Paul EM, et al. (2014). The roles of P2Y2 purinergic receptors in osteoblasts and mechanotransduction. PLoS One. 30; 9: e108417.

You J, Reilly GC, Zhen X, Yellowley CE, et al. (2001). Osteopontin gene regulation by oscillatory fluid flow via intracellular calcium mobilization and activation of mitogen-activated protein kinase in MC3T3-E1 osteoblasts. $J$. Biol. Chem. 276: 13365-13371.http://dx.doi.org/10.1074/jbc.M009846200

You J, Jacobs CR, Steinberg TH and Donahue HJ (2002). P2Y purinoceptors are responsible for oscillatory fluid flowinduced intracellular calcium mobilization in osteoblastic cells. J. Biol. Chem. 277: 48724-48729. http://dx.doi. org/10.1074/jbc.M209245200

Genetics and Molecular Research 16 (1): gmr16019363 